\title{
Qualidade de Organossolo sob diferentes usos antrópicos em áreas de preservação permanente no Distrito Federal ${ }^{1}$
}

\author{
Luciano N. de A. e Moura², Marilusa P. C. Lacerda² \& Maria L. G. Ramos ${ }^{2}$
}

\begin{abstract}
RESUMO
O uso e a ocupação das terras devem ser planejados de forma racional a fim de evitar impactos ambientais negativos nos recursos naturais, particularmente em áreas ambientalmente protegidas por lei. O objetivo deste trabalho foi avaliar atributos de qualidade dos solos em áreas de preservação permanente da Microbacia do Ribeirão do Gama, Distrito Federal, Brasil, para verificar o efeito do uso antrópico. Foram avaliados atributos físicos, químicos e biológicos de qualidade de organossolos em cinco áreas de uso e ocupação diversificada das terras: três áreas cultivadas (hortaliças, eucalipto e mandioca) e duas áreas preservadas (pastagem nativa e mata de galeria). As amostras de solo foram coletadas nas camadas de 0-10 e 10-20 cm. Os dados obtidos pelas análises físicas, químicas e biológicas dos solos foram avaliados estatisticamente por meio do software Sisvar 4.6. Os resultados obtidos mostraram influência dos usos e ocupações diferentes das terras em relação aos atributos de qualidade dos solos. O manejo dos solos adotado nessas áreas foi considerado o principal fator de alteração dos atributos físicos, químicos e biológicos, destacando-se a compactação dos solos sob áreas de vegetação nativa e diminuição da matéria orgânica nos solos em sistemas cultivados.
\end{abstract}

Palavras-chave: manejo do solo, atributos físicos, atributos químicos, atributos biológicos, Ribeirão do Gama

\section{Quality of Histosol under different on anthropic uses in permanent preservation areas in the Distrito Federal}

\begin{abstract}
The use and occupation of soil should be planned in a rational way to avoid negative environmental impacts on natural resources, particularly in areas environmentally protected by law. This study aimed to evaluate quality attributes of soil in permanent preservation areas of a Ribeirão do Gama Watershed, Distrito Federal, Brazil, to verify the influence of anthropic use. The physical, chemical and biological attributes of Histosol quality were assessed in five areas of diversified use and soil occupation: three areas under cultivation (vegetables, eucalyptus and cassava) and two preserved areas (native pasture and gallery forest). Soil samples were collected in 0-10 and $10-20 \mathrm{~cm}$ depth. The data obtained for physical, chemical and biological analysis of the soils were statistically evaluated using the Sisvar 4.6 software. The results obtained showed the influence of different uses and soil occupation in relation to the attributes of soil quality. The adopted management in these areas was considered the main factor of change in the physical, chemical and biological attributes, highlighting the compaction of soils under native vegetation and decrease of organic matter in the soils under cultivated systems.
\end{abstract}

Key words: soil management, physical attributes, chemical attributes, biological attributes, Ribeirão do Gama

${ }^{1}$ Parte da Dissertação de Mestrado do primeiro autor, apresentada à Universidade de Brasília, com projeto financiado pela FAP-DF

${ }^{2}$ Faculdade de Agronomia e Medicina Veterinária/Universidade de Brasília, Campus Universitário Darcy Ribeiro, Instituto Central de Ciências Ala Sul, C.P. 4.508, Asa Norte, Brasília, DF. CEP 70910-960. Fone: (61) 3107-7176/3107-7174. E-mail: lucianomoura26@gmail.com; marilusa@unb.br; lucrecia@unb.br 


\section{INTRODUÇÃO}

O aumento das áreas ocupadas pelas atividades agropecuárias ocorre, muitas vezes, sem planejamento adequado de uso e manejo das terras, desrespeitando o Código Florestal vigente, com desmatamento descontrolado e ocupação de áreas de preservação ambiental. Apesar dos seus esforços, os órgãos fiscalizadores não têm sido suficientes para conter o processo de ocupação desordenada e degradação ambiental, que afetam a qualidade dos solos e dos corpos d'água.

Entre as áreas de preservação ambiental desrespeitadas pelas atividades agropecuárias se destacam as Áreas de Preservação Permanente - APP's (Art. $2^{\circ}$ da Lei 4.771/1965 do Código Florestal (Brasil, 1965) que são áreas com funções específicas de proteção aos cursos d'água, lagoas, lagos ou reservatórios, naturais ou artificiais, que sofrem diretamente os efeitos do uso e a ocupação desordenada das terras. A ocupação dessas áreas provoca, com a retirada da vegetação nativa, efeitos negativos sobre o ecossistema e seus recursos naturais, podendo desencadear impermeabilização do solo e processos erosivos com consequente assoreamento e poluição dos mananciais, conforme constatado por vários pesquisadores, citando-se Nishi et al. (2010).

A degradação ambiental em APP's ocupadas por usos diversificados pode ser realizada por meio de avaliação da qualidade dos solos. Para Karlen et al. (1997) qualidade do solo (QS) é definida como a capacidade do solo em desempenhar sua função dentro dos limites dos ecossistemas naturais ou cultivados para suportar plantas e animais, resistir à erosão e reduzir impactos negativos associados aos recursos água e ar. Segundo esses autores, a QS pode ser medida por meio da quantificação de alguns atributos dos solos, ou seja, de suas propriedades físicas, químicas e biológicas, o que possibilita o monitoramento de mudanças, a médio e longo prazos, no estado de qualidade desses solos.

A mudança da vegetação natural para sistemas de exploração agropecuária provoca alterações profundas nos atributos do solo e a avaliação da QS fornece subsídios para o diagnóstico do grau de desequilíbrio ambiental. Neste contexto, a QS tem sido utilizada para avaliar o manejo dos solos em vários sistemas de cultivo agrícola, assim como em áreas de vegetação nativa convertida em usos antrópicos diversificados (Carneiro et al., 2009)

Seguindo a tendência de desenvolvimento do Brasil, o Distrito Federal (DF) vem sofrendo, nos últimos anos, uma ampliação de áreas destinadas para uso agropecuário, além de intenso processo de urbanização com a ocupação desordenada das terras e o uso impróprio de áreas legalmente preservadas. As APPs estão sendo suprimidas pelo uso urbano e rural prejudicando o equilíbrio ambiental e as condições necessárias para a manutenção da qualidade do solo e dos seres vivos.

Diante deste cenário observado no DF, particularmente na ocupação irracional das APP's, torna-se necessário avaliar o efeito do uso e a ocupação inadequada das terras em cujas áreas podem provocar desequilíbrio ambiental. Assim, o objetivo deste trabalho foi avaliar a qualidade dos solos sob uso diversificado nas áreas de preservação permanente da Microbacia do Ribeirão do Gama, Brasília, DF, a fim de fornecer dados para diagnosticar o impacto ambiental provocado pela ocupação antrópica.

\section{MATERIAL E MÉTODOS}

Este trabalho foi realizado nas áreas de preservação permanente do terço médio do leito Ribeirão do Gama, englobando o Núcleo Rural Vargem Bonita (NRVB) e a Fazenda Água Limpa da Universidade de Brasília (FAL/UnB) em Brasília, DF, em cinco áreas com diferentes usos cujos pontos amostrais foram georreferenciados com GPS Garmin MAP60CSX, plotados no mapa de localização da área e dos pontos amostrais apresentados na Figura 1, descritos a seguir:

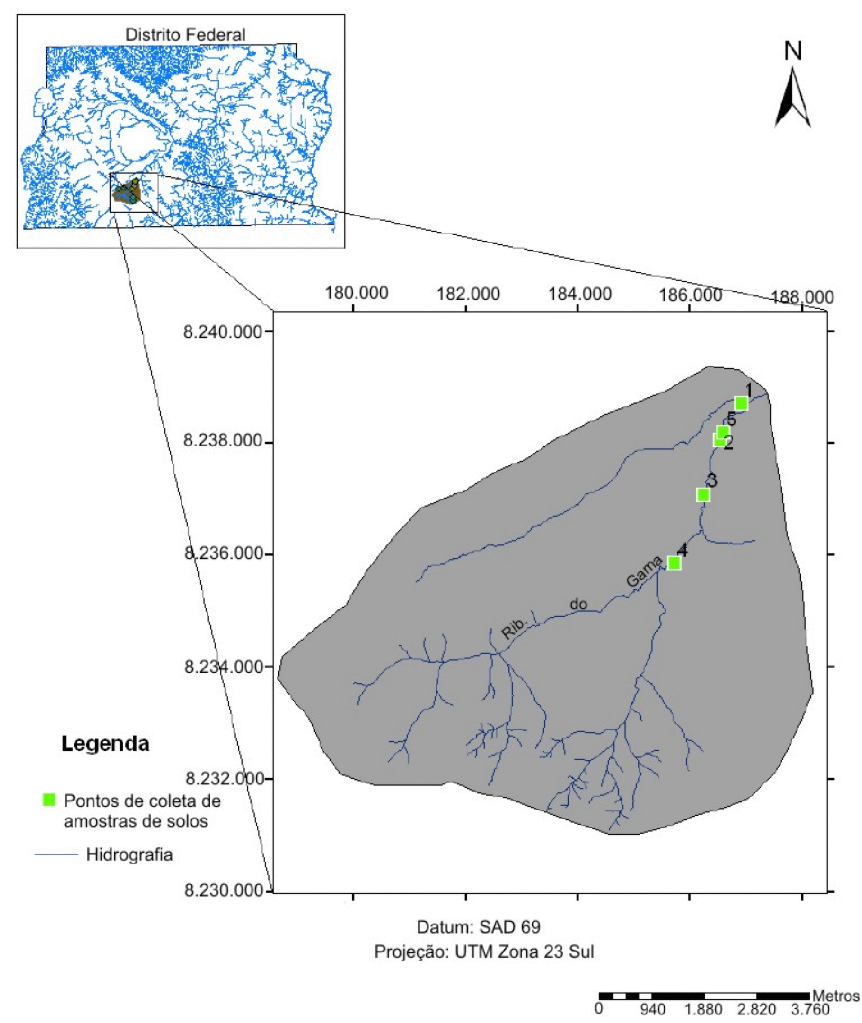

Figura 1. Mapa de localização da microbacia estudada do Ribeirão do Gama no Distrito Federal e dos cinco pontos amostrais

- Ponto 1 - Cultivo de hortaliças folhosas - CH: APP da margem esquerda do Ribeirão do Gama, no NRVB, localizado nas coordenadas UTM (Fuso $23 \mathrm{~S}$ ) $186.911 \mathrm{~m}$ e $8.238 .705 \mathrm{~m}$, com cultivo de hortaliças folhosas sob sistema intensivo de manejo, desde 1980. O solo amostrado foi classificado como um Organossolo Fólico Fíbrico típico, de classe textural francoargilo-siltosa, de acordo com SiBCS (EMBRAPA, 2006);

- Ponto 2 - Cultivo de eucalipto (Eucalyptus spp.) - CE: APP da margem esquerda do Ribeirão do Gama, no NRVB, situado nas coordenadas UTM (Fuso 23 S) $186.533 \mathrm{~m}$ e $8.238 .066 \mathrm{~m}$, com eucalipto sob sistema de cultivo perene, desde 1990, sob Organossolo Fólico Fíbrico típico, de classe textural franca, de acordo com SiBCS (EMBRAPA, 2006);

- Ponto 3 - Cultivo de mandioca (Manihot esculenta Crantz) - CM: APP da margem esquerda do Ribeirão do 
Gama, no NRVB, nas coordenadas UTM (Fuso 23 S) 186.238 $\mathrm{m}$ e $8.237 .082 \mathrm{~m}$, com plantio de mandioca sob sistema semiintensivo de manejo, desde 1980, em Organossolo Fólico Fíbrico típico, de classe textural franco-argilo-siltosa de acordo com SiBCS (EMBRAPA, 2006);

- Ponto 4 - Pastagem nativa - PN: APP da margem direita do Ribeirão do Gama, na Fazenda Água Limpa da Universidade de Brasília - FAL/UnB, localizado nas coordenadas UTM (Fuso $23 \mathrm{~S}$ ) 185.722 e $8.235 .856 \mathrm{~m}$, em que a pastagem nativa não apresenta tratos culturais, utilizada na estação seca para o pastejo de bovinos e equinos, com densidade de ocupação menor que 2 animais ha ${ }^{-1}$. O solo é constituído por Organossolo Fólico Fíbrico típico, de classe textural franco-siltosa, de acordo com SiBCS (EMBRAPA, 2006);

- Ponto 5 - Mata de galeria - MG: APP da margem direita do Ribeirão do Gama, na FAL/UnB, disposto nas coordenadas UTM (Fuso $23 \mathrm{~S}$ ) 186.589 e $8.238 .179 \mathrm{~m}$, sendo caracterizada por uma área preservada de mata de galeria, desenvolvida em Organossolo Fólico Fíbrico típico, de classe textural francosiltosa a argilo-siltosa, de acordo com SiBCS (EMBRAPA, 2006).

Todas as áreas de estudo se localizam na faixa da APP, entre 10 e $25 \mathrm{~m}$ das margens do Ribeirão do Gama. Em cada ponto amostral foram coletadas amostras de solo nas profundidades de $0-10$ e $10-20 \mathrm{~cm}$. As amostras normais foram constituídas por amostras compostas por 5 repetições dispostas em um raio de $10 \mathrm{~m}$, para as duas profundidades avaliadas. As amostras indeformadas foram coletadas por meio de amostrador tipo UHLAND, com uso de cilindros de $100 \mathrm{~cm}^{3}$, com três repetições para cada profundidade estudada.

Os indicadores de qualidade do solo selecionados para o estudo foram densidade do solo (Ds) e estabilidade de agregados (EA); entre os físicos; capacidade de troca catiônica (CTC), matéria orgânica do solo (MO), entre os químicos; carbono da biomassa microbiana $\left(\mathrm{C}_{\mathrm{BMS}}\right)$, carbono orgânico $\left(\mathrm{C}_{\text {org }}\right)$, quociente microbiano (qMIC), nitrogênio da biomassa microbiana $\left(\mathrm{N}_{\mathrm{BMS}}\right)$, nitrogênio total $(\mathrm{Nt})$, respiração basal $(\mathrm{Rb})$ e quociente metabólico $\left(\mathrm{qCO}_{2}\right)$ entre os indicadores biológicos.

A análise laboratorial da densidade do solo (Ds), estabilidade de agregados (EA) e da CTC, foram obtidos segundo metodologias propostas em EMBRAPA (1997).

A matéria orgânica do solo (MO), o carbono orgânico $\left(\mathrm{C}_{\text {org }}\right)$ e o Nt do solo foram quantificados segundo EMBRAPA (1997). $\mathrm{O} \mathrm{C}_{\mathrm{BMS}}$ foi avaliado pelo método da fumigação/extração, de acordo com a metodologia proposta por Vance et al. (1987) e o quociente microbiano (qMIC) foi calculado pela relação entre o $\mathrm{C}_{\mathrm{BMS}}$ e o $\mathrm{C}_{\text {org }}$. Para a determinação do $\mathrm{N}_{\mathrm{BMS}}$ utilizou-se o método proposto por Brookes et al. (1985) e a relação $\mathrm{N}_{\mathrm{BMS}}: \mathrm{Nt}$ foi calculada pela razão entre o $\mathrm{N}_{\mathrm{BMS}}$ e o Nt. A respiração basal $(\mathrm{Rb})$ foi calculada pela quantidade de $\mathrm{C}-\mathrm{CO}_{2}$ liberado nas amostras de solo utilizando-se a metodologia proposta por Alef \& Nannipieri (1995) e o quociente metabólico $\left(\mathrm{qCO}_{2)}\right.$ foi calculado pela razão entre a $\mathrm{Rb}$ e o $\mathrm{C}_{\mathrm{BMS}}$. As análises estatísticas foram realizadas por meio do software estatístico Sisvar, versão 4.6 (Ferreira, 2003) que constituíram de análise de variância (ANOVA) em relação aos 5 tratamentos $(\mathrm{CH}$, $\mathrm{CE}, \mathrm{CM}, \mathrm{PN}$ e MG), 2 profundidades $(0-10$ e $10-20 \mathrm{~cm})$ e 3 repetições, além da interação tratamento* profundidade, seguida pelo teste de média de Tukey a $5 \%$ de probabilidade. Quando a ANOVA não foi considerada significativa em relação à interação tratamento*profundidade, $\mathrm{o}$ teste $\mathrm{t}$ foi realizado a partir das médias dos atributos avaliados em cada uma das áreas amostrais.

\section{RESULTADOS E DISCUSSÃO}

A Tabela 1 apresenta os resultados obtidos para densidade do solo e estabilidade de agregados dos cinco tratamentos avaliados. A densidade do solo da área de pastagem nativa (PN) apresentou o maior valor nas duas profundidades amostradas $\left(1,68 \mathrm{~g} \mathrm{~cm}^{-3}\right)$; já o solo da área com cultivo de eucalipto (CE) mostrou a menor Ds nas duas profundidades estudadas.

Tabela 1. Densidade do solo e estabilidade dos agregados dos solos avaliados em áreas de preservação permanente (APP) da microbacia estudada do Ribeirão do Gama

\begin{tabular}{lccc}
\hline \multirow{2}{*}{ Tratamento } & \multicolumn{3}{c}{ Profundidade (cm) } \\
\cline { 2 - 4 } & $\mathbf{0 - 1 0}$ & $\mathbf{1 0 - 2 0}$ & Média \\
Hortaliças (CH) & \multicolumn{2}{c}{ Densidade do solo - Ds $\left(\mathrm{g} \mathrm{cm}^{-3}\right)$} \\
Eucalipto (CE) & 1,01 & 0,95 & $0,98 \mathrm{bc}$ \\
Mandioca (CM) & 0,84 & 0,82 & $0,83 \mathrm{C}$ \\
Pastagem Nativa (PN) & 1,23 & 1,17 & $1,20 \mathrm{~b}$ \\
Mata de Galeria (MG) & 1,68 & 1,68 & $1,68 \mathrm{a}$ \\
Média & 1,31 & 1,22 & $1,26 \mathrm{~b}$ \\
\hline & $1,21 \mathrm{~A}$ & $1,16 \mathrm{~A}$ \\
Hortaliças (CH) & Estabilidade dos agregados do solo - DMP (mm) \\
Eucalipto (CE) & 2,84 & 2,59 & $2,72 \mathrm{a}$ \\
Mandioca (CM) & 2,77 & 2,71 & $2,74 \mathrm{a}$ \\
Pastagem Nativa (PN) & 2,84 & 2,78 & $2,81 \mathrm{a}$ \\
Mata de Galeria (MG) & 2,54 & 2,39 & $2,47 \mathrm{~b}$ \\
Média & 2,78 & 2,64 & $2,71 \mathrm{a}$ \\
\hline
\end{tabular}

Médias seguidas pela mesma letra maiúscula nas linhas e minúscula nas colunas, não diferem entre si pelo teste t a 0,05 de probabilidade

Segundo Letey (1985) solos que apresentam Ds ao redor de $1,3 \mathrm{~g} \mathrm{~cm}^{-3}$ podem ser considerados ideais para o crescimento da maioria das culturas uma vez não restringem o desenvolvimento radicular. Solos com teor muito alto de matéria orgânica (MO) como é o caso dos solos estudados, Organossolos, são passíveis de apresentar densidades menores que $1 \mathrm{~g} \mathrm{~cm}^{-3}$.

Os sistemas de $\mathrm{PN}$ e mata de galeria (MG) apresentaram valores superiores a $1,2 \mathrm{~g} \mathrm{~cm}^{-3}$. Como a área é ocupada com pastoreio de bovinos durante a seca, o pisoteio associado à textura silto-argilosa, predominante, além da estrutura frágil desses solos, pode ser um dos fatores relacionados ao aumento da Ds. Os pontos avaliados se encontram dentro da APP do Ribeirão do Gama, cuja água é utilizada para dessedentação dos animais durante o pastoreio, além de oferecer proteção contra o calor nas horas mais quentes do dia.

Assim, apenas o sistema PN, com Ds de 1,68 $\mathrm{g} \mathrm{cm}^{-3}$, apresentou a qualidade física do solo comprometida em relação à Ds. O sistema $\mathrm{MG}$ e o cultivo de mandioca apresentaram valores próximos a $1,2 \mathrm{~g} \mathrm{~cm}^{-3}$, o que determina maior atenção para as atividades de pastoreio de bovinos desenvolvidas sobre a área com mata de galeria além de condições de manejo adotadas na área com CM, podendo evoluir com a compactação dos solos.

Segundo Araújo et al. (2007) a Ds diminui com o incremento do teor de $\mathrm{MO}$ e tende a aumentar com a profundidade, sendo 
influenciada por uma infinidade de fatores associados à diminuição do teor de MO, tais como menor agregação, maior compactação e diminuição da porosidade do solo, entre outros. Tal fato não foi significativo nos Organossolos das cinco áreas estudadas com usos diversificados, cuja distribuição de MO ao longo das profundidades avaliadas, foi considerada semelhante (Tabela 2).

Tabela 2. Capacidade de troca catiônica e matéria orgânica dos solos avaliados em áreas de preservação permanente (APP) da microbacia estudada do Ribeirão do Gama

\begin{tabular}{lccc}
\hline \multirow{2}{*}{ Tratamento } & \multicolumn{3}{c}{ Profundidade (cm) } \\
\cline { 2 - 4 } & $\mathbf{0 - 1 0}$ & $\mathbf{1 0 - 2 0}$ & Média \\
Hortaliças (CH) & \multicolumn{1}{c}{ Capacidade } & troca catiônica- CTC $\left(\mathrm{cmol}_{\mathrm{c}} \mathrm{dm}^{-3}\right)$ \\
Eucalipto (CE) & 18,20 & 17,57 & $17,88 \mathrm{a}$ \\
Mandioca (CM) & 17,17 & 15,87 & $16,52 \mathrm{a}$ \\
Pastagem Nativa (PN) & 21,67 & 21,83 & $21,75 \mathrm{a}$ \\
Mata de Galeria (MG) & 18,07 & 17,57 & $17,82 \mathrm{a}$ \\
Média & 19,50 & 16,00 & $17,75 \mathrm{a}$ \\
\hline \multicolumn{4}{c}{ Matéria orgânica do solo $-\mathrm{MO}\left(\mathrm{g} \mathrm{kg}^{-1}\right)$} \\
Hortaliças (CH) & $18,91 \mathrm{~A}$ & $17,80 \mathrm{~B}$ \\
Eucalipto (CE) & $11,3 \mathrm{CA}$ & $115,0 \mathrm{cdA}$ \\
Mandioca (CM) & $99,0 \mathrm{cA}$ & $93,7 \mathrm{dA}$ \\
Pastagem Nativa (PN) & $122,3 \mathrm{cA}$ & $126,0 \mathrm{bcA}$ \\
Mata de Galeria (MG) & $261,7 \mathrm{aA}$ & $264,3 \mathrm{aA}$ \\
\hline
\end{tabular}

Médias seguidas pela mesma letra maiúscula nas linhas e minúscula nas colunas não diferem entre si pelo teste $t$ a 0,05 de probabilidade

Valladares (2008) encontrou, analisando Organossolos da Bahia, BA, correlação negativa entre a Ds e a MO, o que pode ser explicado pela baixa densidade da matéria orgânica em relação à fração mineral do solo; já Bertol et al. (2006) constataram o aumento da densidade do solo quando a preparação do solo adota uso de máquinas e elevada carga animal, o que foi verificado nas PN, MG e CM.

Assim, com relação ao indicador Ds os solos das áreas sob cultivo de hortaliças e eucalipto se encontram em condições de sustentabilidade. A diferença verificada entre os solos das duas áreas ocupadas por agricultura, com cultivo de mandioca e de hortaliças, está relacionado, provavelmente, ao manejo e ao uso mais intensivo da área com CM.

Em todos os tratamentos avaliados o DMP dos agregados dos solos diminuiu com a profundidade (Tabela 1) e foi semelhante em todas as cinco áreas estudadas, com exceção da área sob PN, que apresentou o menor valor de DMP, de 2,47 $\mathrm{mm}$. Este comportamento também pode estar relacionado ao pisoteio dos animais, à textura desse solo e ao alto valor de Ds constatado na PN. Os valores obtidos para a DMP dos agregados dos Organossolos estudados são considerados altos quando comparado a outros solos, uma vez que a MO é considerada por muitos pesquisadores como o principal agente de estabilização de agregados (Gang et al., 1998).

Para Moreira \& Siqueira (2006) uma boa estrutura para o desenvolvimento vegetal e a sustentabilidade ambiental, depende da presença de agregados estáveis com diâmetro maior que $1 \mathrm{~mm}$, o que permite considerar que os solos dos tratamentos avaliados se encontram em condições de sutentabilidade ambiental em relação ao atributo estabilidade de agregados dos solos.

Os resultados obtidos para os indicadores químicos de qualidade do solo avaliados (capacidade de troca catiônica e matéria orgânica do solo) estão dispostos na Tabela 2. A CTC apresentou valores semelhantes em todos os sistemas avaliados, com valor médio entre 16,52 na área sob eucalipto e 21,75 $\mathrm{cmol}_{\mathrm{c}} \mathrm{dm}^{-3}$ na área sob mandioca. A CTC de Organossolos nas camadas superficiais do solo, pode variar de 22,8 a $68,8 \mathrm{cmol}_{\mathrm{c}}$ $\mathrm{dm}^{-3}$ entre $0-10 \mathrm{~cm}$, a 39,7 a $120,1 \mathrm{cmol}_{\mathrm{c}} \mathrm{dm}^{-3}$ entre $10-20 \mathrm{~cm}$ e esta variação pode ser devida à drenagem e à altitude das áreas sob estudo (Silva et al., 2009).

Segundo Moreira \& Siqueira (2006) em áreas cultivadas a CTC pode ser alterada nas camadas superficiais do solo pelo efeito da calagem utilizada na correção do solo, além da adição de restos culturais; já em áreas preservadas a CTC é influenciada pelo teor de MO.

Os solos avaliados, Organossolos Fólicos fíbricos, são solos geneticamente enriquecidos em MO mas se sabe que o manejo do solo pode alterar os teores de MO no solo (Perez et al., 2004). A análise estatística dos resultados obtidos para os teores de matéria orgânica (MO) apresentou interação significativa entre os tratamentos e as profundidades avaliadas. As áreas sob $\mathrm{CH}, \mathrm{CE}$ e CM apresentaram os menores valores nas duas profundidades avaliadas e a área sob PN sinalizou os maiores valores, de 261,7 e 264,3 $\mathrm{g} \mathrm{kg}^{-1}$, respectivamente, para as profundidades estudadas, enquanto os solos sob mata de galeria apresentaram valores intermediários de 205,0 e 150,0 $\mathrm{g} \mathrm{kg}^{-1}$, respectivamente, nas profundidades de 0-10 e 10-20 cm (Tabela 2).

O solo sob MG foi o único sistema que apresentou diferença estatística significativa dos valores de MO nos solos nas duas profundidades avaliadas, havendo redução no teor de $\mathrm{MO}$ com o aumento da profundidade de $55,0 \mathrm{~g} \mathrm{~kg}^{-1}$. Nos demais tratamentos os valores de MO permaneceram semelhantes nas duas profundidades avaliadas. A elevada taxa de deposição de MO na camada superficial dos solos sob MG e a lenta decomposição pode justificar este comportamento.

Os valores mais altos obtidos de MO foram na pastagem nativa e mata de galeria, que constituem sistemas conservadores de MO. Em solos sob vegetação natural, tal como na PN e MG, a preservação de MO tende a ser elevada pois o revolvimento do solo é mínimo sendo o aporte de carbono nas matas mais elevado do que em áreas cultivadas (Perez et al., 2004).

Já em áreas cultivadas, que é o caso dos tratamentos $\mathrm{CH}$, $\mathrm{CE}$ e $\mathrm{CM}$, os teores de $\mathrm{MO}$ diminuem em relação aos valores obtidos na PN e MG já que as frações orgânicas são mais expostas ao ataque de micro-organismos em função do maior revolvimento e desestruturação do solo. As taxas de perda de MO são afetadas, sobremaneira, pela drenagem e preparo do solo, especialmente pela intensidade do revolvimento, que influencia a temperatura, umidade, aeração do solo, o grau de fracionamento e incorporação dos resíduos culturais (Moreira \& Siqueira, 2006).

Em um trabalho avaliando Organossolos de diferentes regiões do Brasil, Ebeling et al. (2011) obtiveram valores de MO variando entre 116,66 e 745,83 $\mathrm{g} \mathrm{kg}^{-1}$, no qual os autores atribuem esta variabilidade às condições ambientais dos locais de amostragem, além da heterogeneidade das camadas de depósitos orgânicos, o que reflete nas variações da cobertura vegetal, umidade e outros fatores relacionados. 
A biomassa microbiana é a fração viva da MO e é afetada diretamente por fatores ambientais, como o manejo do solo (Perez et al., 2004) e a fertilização (Coser et al., 2007), sendo bastante sensível às alterações que ocorrem no manejo (Cardoso et al., 2009)

Os resultados obtidos para os atributos biológicos de qualidade do solo avaliados se encontram na Tabela 3. Em relação ao carbono da biomassa microbiana o maior valor de $\mathrm{C}_{\mathrm{BMS}}$ foi verificado nas áreas sob $\mathrm{PN}$, obtendo 910,14 mg C $\mathrm{kg}^{-1}$ e 733,2 $\mathrm{mg} \mathrm{C} \mathrm{kg}^{-1}$, respectivamente, nas profundidades de 0-10 e 10-20 cm, com diferença significativa em relação aos valores obtidos para os solos analisados dos demais tratamentos. Os menores valores foram encontrados nas áreas sob $\mathrm{CH}, \mathrm{CE}$ e $\mathrm{MG}$, com valores médios das profundidades estudadas de 157,$78 ; 186,06 ; 158,08 \mathrm{mg} \mathrm{C} \mathrm{kg}^{-1}$, respectivamente. O cultivo de mandioca apresentou um valor intermediário em relação aos demais, com valor médio nas profundidades avaliadas, de $314,70 \mathrm{mg} \mathrm{C} \mathrm{kg}^{-1}$.

$\mathrm{O}$ valor mais alto de $\mathrm{C}_{\mathrm{BMS}}$ foi verificado na pastagem nativa. Alguns autores também observaram altos valores de $\mathrm{C}_{\mathrm{BMS}} \mathrm{em}$ áreas sob pastagem, tal como Araújo et al. (2007) que constaram valor de $\mathrm{C}_{\mathrm{BMS}}$ dos Latossolos estudados na área de pastagem

Tabela 3. Atributos biológicos dos solos avaliados em áreas de preservação permanente (APP) da microbacia estudada do Ribeirão do Gama

\begin{tabular}{|c|c|c|c|}
\hline \multirow{2}{*}{ Tratamento } & \multicolumn{3}{|c|}{ Profundidade (cm) } \\
\hline & $0-10$ & $11-20$ & Média \\
\hline \multicolumn{4}{|c|}{ Carbono da biomassa microbiana - $\mathrm{C}_{\mathrm{BMS}}-\left(\mathrm{mg} \mathrm{C} \mathrm{kg}{ }^{-1}\right.$ de solo $)$} \\
\hline Hortaliças (CH) & 188,21 & 127,35 & $157,78 \mathrm{c}$ \\
\hline Eucalipto (CE) & 204,59 & 167,54 & $186,06 \mathrm{c}$ \\
\hline Mandioca (CM) & 324,83 & 304,56 & $314,70 \mathrm{~b}$ \\
\hline Pastagem nativa (PN) & 910,14 & 733,20 & $821,67 \mathrm{a}$ \\
\hline Mata de galeria (MG) & 183,77 & 132,39 & $158,08 \mathrm{c}$ \\
\hline Média & $362,31 \mathrm{~A}$ & $293,00 \mathrm{~B}$ & \\
\hline \multicolumn{4}{|c|}{ Respiração basal do dolo - $\mathrm{Rb}-\left(\mathrm{mg} \mathrm{C}-\mathrm{CO}_{2} \mathrm{~kg}^{-1}\right.$ de solo $\left.\mathrm{d}^{-1}\right)$} \\
\hline Hortaliças $(\mathrm{CH})$ & $98,28 \mathrm{cA}$ & $91,72 \mathrm{dA}$ & \\
\hline Eucalipto (CE) & $91,45 \mathrm{cA}$ & $88,44 \mathrm{dA}$ & \\
\hline Mandioca (CM) & $256,63 \mathrm{aA}$ & $184,32 \mathrm{bB}$ & \\
\hline Pastagem nativa (PN) & $250,76 \mathrm{aA}$ & $260,60 \mathrm{aA}$ & \\
\hline Mata de galeria (MG) & $156,73 \mathrm{bA}$ & $142,49 \mathrm{cA}$ & \\
\hline \multicolumn{4}{|c|}{ Nitrogênio da biomassa microbiana do solo $-\mathrm{N}_{\mathrm{BMS}}\left(\mathrm{mg} \mathrm{N} \mathrm{kg}^{-1}\right.$ de solo) } \\
\hline Hortaliças (CH) & 22,66 & 17,40 & $20,03 \mathrm{c}$ \\
\hline Eucalipto (CE) & 44,00 & 28,51 & $36,25 \mathrm{bc}$ \\
\hline Mandioca (CM) & 51,47 & 37,35 & $44,41 \mathrm{~b}$ \\
\hline Pastagem nativa (PN) & 108,47 & 72,39 & $90,43 \mathrm{a}$ \\
\hline Mata de galeria (MG) & 38,74 & 18,62 & $28,68 \mathrm{bc}$ \\
\hline Média & $53,06 \mathrm{~A}$ & $34,85 \mathrm{~B}$ & \\
\hline \multicolumn{4}{|c|}{ Nitrogênio total - Nt $\left(\mathrm{g} \mathrm{kg}^{-1}\right)$} \\
\hline Hortaliças $(\mathrm{CH})$ & 3,53 & 2,97 & $3,25 \mathrm{bc}$ \\
\hline Eucalipto (CE) & 2,50 & 2,43 & $2,46 \mathrm{c}$ \\
\hline Mandioca (CM) & 4,46 & 4,09 & $4,28 a b$ \\
\hline Pastagem nativa (PN) & 3,85 & 2,93 & $3,39 \mathrm{bc}$ \\
\hline Mata de galeria (MG) & 4,95 & 5,29 & $5,12 \mathrm{a}$ \\
\hline Média & $3,85 \mathrm{~A}$ & $3,54 \mathrm{~A}$ & \\
\hline \multicolumn{4}{|c|}{ 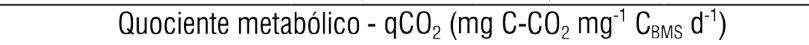 } \\
\hline Hortaliças $(\mathrm{CH})$ & 0,57 & 0,80 & $0,69 \mathrm{~b}$ \\
\hline Eucalipto (CE) & 0,45 & 0,58 & $0,52 \mathrm{bc}$ \\
\hline Mandioca (CM) & 0,85 & 0,62 & $0,74 \mathrm{~b}$ \\
\hline Pastagem Nativa (PN) & 0,29 & 0,36 & $0,33 \mathrm{c}$ \\
\hline Mata de Galeria (MG) & 0,98 & 1,13 & $1,06 \mathrm{a}$ \\
\hline Média & $0,63 \mathrm{~A}$ & $0,70 \mathrm{~A}$ & \\
\hline
\end{tabular}

Médias seguidas pela mesma letra maiúscula nas linhas e minúscula nas colunas, não diferem entre si pelo teste de Tukey a 0,05 de probabilidade semelhantes aos dos Latossolos avaliados sob Cerrado Nativo atribuindo os resultados obtidos ao intenso desenvolvimento do sistema radicular das gramíneas forrageiras, o que favorece a atividade biológica do solo. Carneiro et al. (2009) concordam que o sistema radicular das gramíneas promove a atividade biológica nos solos, uma vez que permite maior entrada de carbono no solo.

Silva et al. (2009) também obtiveram, trabalhando em áreas não antropizadas, maiores valores de $\mathrm{C}_{\mathrm{BMS}} \mathrm{em}$ áreas de cerrado sob Organossolos, entre 185,8 a 294,9 e 429,8 a 551,6 mg C $\mathrm{kg}^{-1}$, nas profundidades de 0-10 e 10-20 cm, respectivamente, e atribuíram esses resultados aos elevados teores de $\mathrm{MO}$ e à má drenagem do solo, que dificulta a decomposição vegetal.

Os maiores valores para a respiração basal $(\mathrm{Rb})$ foram encontrados nos solos sob PN e CM (Tabela 3) nas duas profundidades estudadas $\left(250,76\right.$ e $260,60 \mathrm{mg}$ de $\mathrm{C}-\mathrm{CO}_{2} \mathrm{~kg}^{-1}$ de solo $\mathrm{d}^{-1}$ e 256,63 e 184,32 mg de $\mathrm{C}-\mathrm{CO}_{2} \mathrm{~kg}^{-1}$ de solo $\mathrm{d}^{-1}$, respectivamente) que apresentaram valores semelhantes na profundidade de $0-10 \mathrm{~cm}$. Os menores valores de $\mathrm{Rb}$ foram verificados nos solos sob $\mathrm{CE}$ e $\mathrm{CH}(91,45$ e $88,44 \mathrm{mg}$ de $\mathrm{C}-\mathrm{CO}_{2} \mathrm{~kg}^{-1}$ de solo d ${ }^{-1}$ e 98,28 e 91,72 mg de C-CO $\mathrm{kg}^{-1}$ de solo $\mathrm{d}^{-1}$, respectivamente nas profundidades de 0-10 e 10-20 $\mathrm{cm}$ ) apresentando-se semelhantes em ambas as profundidades avaliadas.

$\mathrm{A} \mathrm{Rb}$ do solo da mata de galeria apresentou valores intermediários nas duas profundidades estudadas em relação aos demais (156,73 e 142,49 $72 \mathrm{mg}$ de $\mathrm{C}-\mathrm{CO}_{2} \mathrm{~kg}^{-1}$ de solo d ${ }^{-1}$, respectivamente para as profundidades de 0-10 e 10-20 cm).

Os menores teores de $\mathrm{Rb}$ observados nos solos sob $\mathrm{CE}$ e $\mathrm{CH}$ nas duas profundidades, podem ser explicados pela menor atividade da microbiota em sistemas cultivados visto que promovem o revolvimento e a maior exposição do solo. $\mathrm{O}$ manejo não conservacionista adotado nesses solos pode reduzir a quantidade de micro-organismos no solo e, consequentemente, a $\mathrm{Rb}$ dos solos, uma vez que a $\mathrm{Rb}$ representa a oxidação da $\mathrm{MO}$ por organismos aeróbios do solo que utilizam o $\mathrm{O}_{2}$ como aceptor final de elétrons passível de ser avaliada tanto pelo consumo de $\mathrm{O}_{2}$ como pela produção de $\mathrm{CO}_{2}$ (Moreira \& Siqueira, 2006).

Os dados de Rb obtidos no presente trabalho são, em geral, superiores aos apresentados por Silva et al. (2009) em áreas preservadas sob Organossolos, com valores entre 27,69 e 61,43 $\mathrm{mg}$ de $\mathrm{C}-\mathrm{CO}_{2} \mathrm{~kg}^{-1}$ de solo $\mathrm{d}^{-1}$.

Observou-se correspondência entre os valores obtidos de carbono da biomassa microbiana com os resultados encontrados para a respiração basal do solo, destacando-se os solos sob PN, $\mathrm{CH}$ e $\mathrm{CE}$.

Uma respiração basal maior do solo, geralmente é resultado de maior atividade biológica e está associada à matéria orgânica lábil, principalmente do consumo de carbono oxidável para a manutenção da população microbiana indicando, a curto prazo, maior liberação de nutrientes às plantas e, a longo prazo, perda de carbono do solo para a atmosfera (Follet \& Schimel, 1989).

Conforme os resultados obtidos para nitrogênio da biomassa microbiana $\left(\mathrm{N}_{\text {BMS }}\right)$ dos solos estudados (Tabela 3) o maior valor foi constatado no solo sob $\mathrm{PN}$, com valor médio nas profundidades avaliadas de $90,43 \mathrm{mg}^{\mathrm{de}} \mathrm{N} \mathrm{kg}^{-1}$. O maior valor de $\mathrm{N}_{\mathrm{BMS}}$ em áreas sob pastagem pode ser devido à excreção dos animais na área (Garcia \& Nahas, 2007). Os menores valores 
foram observados no solo sob $\mathrm{CH}$ (valor médio de $20,03 \mathrm{mg}$ de $\mathrm{N} \mathrm{kg}^{-1}$ de solo nas profundidades de 0-10 e 10-20 cm) considerados semelhantes aos valores obtidos nos solos sob CE e MG. O solo sob CM apresentou valor de $\mathrm{N}_{\text {BMS }}$ semelhante ao CE. Assim, os sistemas cultivados (CH, CE e CM) apresentaram os menores valores de $\mathrm{N}_{\text {BMS }}$ uma vez que, provavelmente, o revolvimento do solo utilizado no manejo, particularmente da $\mathrm{CH}$ e CM, estimula o desenvolvimento de micro-organismos e os processos oxidativos do solo (Breland \& Eltun, 1999).

Os valores de $\mathrm{N}_{\mathrm{BMS}}$ foram menores na camada de 10-20 nos solos dos tratamentos estudados, com exceção de MG. Os maiores valores de $\mathrm{N}_{\text {BMS }}$ nas camadas superficiais estão de acordo com os resultados obtidos por Coser et al. (2007) e Perez et al. (2004) que verificaram diminuição dos valores da $\mathrm{N}_{\text {BMS }}$ com o aumento da profundidade. A transformação do nitrogênio orgânico encontrado nos horizontes superficiais, em determinado período, é influenciada pelos fatores que controlam o crescimento e atividade microbiana no solo, como a natureza dos resíduos, a temperatura, o pH, umidade e a aeração (Dao, 1998).

Em relação às profundidades avaliadas, os valores de nitrogênio total $(\mathrm{Nt})$ variaram conforme os resultados apresentados na Tabela 3. O maior valor de $\mathrm{Nt}$ foi observado no solo estudado do tratamento MG $\left(4,95\right.$ e $5,29 \mathrm{~g} \mathrm{~kg}^{-1}$ nas profundidades de $0-10$ e $10-20 \mathrm{~cm}$, respectivamente) que apresentou diferença significativa em relação aos resultados de Nt dos solos dos tratamentos CH, CE e PN que, por sua vez, apresentaram os valores mais baixos de $\mathrm{Nt}$ nos solos analisados (valores médios em relação às profundidades avaliadas de 3,25 , 2,46 e $3,39 \mathrm{~g} \mathrm{~kg}^{-1}$, respectivamente ). No solo sob CM o valor obtido de $\mathrm{Nt}$ (valor médio de $4,28 \mathrm{~g} \mathrm{~kg}^{-1}$ nas profundidades estudadas) foi considerado semelhante aos valores de Nt dos solos sob CH, PN e MG.

Os valores obtidos para $\mathrm{Nt}$ dos solos sob os sistemas cultivados, particularmente $\mathrm{CM} \mathrm{e} \mathrm{CH}$, semelhantes aos valores encontrados aos sistemas preservados (PN e MG) podem estar relacionados à fertilização adotada nos tratamentos cultivados uma vez que a adubação nitrogenada promove aumento nos estoques de Nt (Weber \& Mielniczuk, 2009).

$\mathrm{O}$ valor de Nt obtido para os solos sob PN foi considerado baixo para sistemas considerados preservados concordando com o trabalho de Azevedo \& Sverzut (2007) ao verificarem que o uso do solo com pastagem reduziu o teor de nitrogênio no solo, devido à exportação de nutrientes provocada pelo pastejo. Weber \& Mielniczuk (2009) relatam que como o acúmulo de Nt no solo ocorre lentamente, são necessários experimentos de longa duração para a observação do impacto de práticas de manejo sobre a disponibilidade e o acúmulo desse nutriente no solo e em áreas não perturbadas, como na $\mathrm{MG}$, em virtude do acúmulo de restos vegetais na superfície e o preparo do solo com aração e gradagem, que promovem aumento da mineralização de nutrientes, dentre eles o nitrogênio (Breland \& Eltun, 1999).

Os dados obtidos para o quociente metabólico $\left(\mathrm{qCO}_{2}\right)$ dos solos estudados sob usos e ocupações diversificadas, são apresentados na Tabela 3. O maior valor foi verificado no solo da MG (0,98 e $1,13 \mathrm{mg}$ de C-CO $\mathrm{mg}^{-1} \mathrm{C}_{\text {BMS }} \mathrm{d}^{-1}$ nas profundidades de 0-10 e 10-20 cm, respectivamente) distinto dos resultados encontrados nos solos dos demais tratamentos. Na mata de galeria ocorre maior incorporação de resíduos orgânicos no solo em relação aos demais tratamentos, que aumenta o $\mathrm{qCO}_{2}$ (Ocio \& Brookes, 1990). Os maiores valores de $\mathrm{qCO}_{2}$ indicam que a população microbiana possui alto gasto de energia na sua manutenção, mediante altas taxas de respiração (Dias Júnior et al., 1998).

O menor valor de $\mathrm{qCO}_{2}$ foi observado no solo da $\mathrm{PN}(0,29 \mathrm{e}$ $0,36 \mathrm{mg}$ de $\mathrm{C}-\mathrm{CO}_{2} \mathrm{mg}$ de C-CO $\mathrm{mg}^{-1} \mathrm{C}_{\mathrm{BMS}} \mathrm{d}^{-1}$, nas profundidades de 0-10 e 10-20 cm, respectivamente) considerado semelhante em relação aos resultados de $\mathrm{qCO}_{2}$ do solo sob CE. Para Insam \& Domsch (1988) a respiração microbiana por unidade de biomassa microbiana diminui em sistemas mais estáveis, tais como os tratamentos PN e CE. Os resultados de $\mathrm{qCO}_{2}$ dos solos sob $\mathrm{CH}, \mathrm{CE}$ e CM não apresentaram diferenças significativas entre si.

\section{ConclusõES}

1. Os indicadores físicos de qualidade do solo mostram acréscimo da densidade dos solos nas áreas de pastagem nativa e mata de galeria.

2. Os teores de MO mostram altos valores nos solos das áreas de vegetação nativa (mata de galeria e pastagem nativa).

3. Há atividade microbiana mais intensa nas áreas de pastagem nativa, cultivo mandioca e mata de galeria e maior biomassa microbiana na pastagem nativa.

\section{LITERATURA CITADA}

Alef, K.; Nannipieri, P. Methods in applied soil microbiology and biochemistry. London: Academic Press, 1995. 576p.

Araújo, R.; Goedert, W. J.; Lacerda; M. P. C. Qualidade de um solo sob diferentes usos e sob cerrado nativo. Revista Brasileira de Ciência do Solo, v.31, p.1099-1108, 2007.

Azevedo, E. C.; Sverzut, C. B. Alterações dos atributos físicos e químicos do solo sob pastagem no sudoeste do Estado de Mato Grosso. Edufmt. Cuiabá/MT. Revista Agricultura Tropical, v.9, p.7-23, 2007.

Bertol, I.; Mafra, A. L.; Cogo, N. P. Conservação do solo em pastagens. Pedreira, C. G. S.; Moura, J. C.; Silva, S. C.; Faria, V. P. (ed.) As pastagens e o meio ambiente. Simpósio sobre Manejo da Pastagem, 23, 2006. Piracicaba. Anais... Piracicaba: FEALQ, 2006. p.139-163.

Brasil. Lei n. 4771 de 15 de setembro de 1965. Institui o novo Código Florestal. Diário Oficial da República Federativa do Brasil, Brasília, 16 de setembro de 1965. http://www.planalto.gov.br/ccivil_03/Leis/L4771.htm. 03 Jun. 2011.

Breland, T. A.; Eltun, R. Soil microbial biomass and mineralization of carbon and nitrogen in ecological, integrated and conventional forage and arable cropping systems. Biology and Fertility of Soils, v.30, p.193-201, 1999.

Brookes, P. C.; Landman, A.; Pruden, G.; Jenkinson, D. S. Chloroform fumigation and the release of soil nitrogen: a rapid direct extraction method to measure microbial biomass nitrogen in soil. Soil Biology and Biochemistry, v.17, p.837-842, 1985. 
Cardoso, E. L. Silva, M. L. N.; Moreira, F. M. de S.; Curi, $\mathrm{N}$. Atributos biológicos indicadores da qualidade do solo em pastagem cultivada e nativa no pantanal. Pesquisa Agropecuária Brasileira, v.44, p.631-637, 2009.

Carneiro, M. A. C.; Souza, E. C.; Reis, D. F.; Pereira, H. S.; Azevedo, W. R. Atributos físicos, químicos e biológicos de solo de Cerrado sob diferentes sistemas de uso e manejo. Revista Brasileira de Ciência do Solo, v.33, p.147-157, 2009.

Coser, T. R.; Ramos, M. L. G.; Amabile, R. F.; Ribeiro Junior, W. Q. Nitrogênio da biomassa microbiana em solo de Cerrado com aplicação de fertilizante nitrogenado. Pesquisa Agropecuária Brasileira, v.42, p.399-406, 2007.

Dao, T. H. Tillage and crop residue effects on carbon dioxide evolution and carbon storage in a Paleustoll. Soil Science Society of American Journal, v.62, p.250-256, 1998.

Dias Júnior, H. E.; Moreira, F. M. S.; Siqueira, J. O.; Silva, R. Metais pesados, densidade e atividade microbiana em solo contaminado por rejeitos de indústria de zinco. Revista Brasileira de Ciência do Solo, v.22, p.631-640, 1998.

Ebeling, A. G.; Anjos, L. H. C. dos; Perez, D. V.; Pereira, M. G.; Gomes, F. W. de F. Atributos químicos, carbono orgânico e substâncias húmicas em Organossolos háplicos de várias regiões do Brasil. Revista Brasileira de Ciência do Solo, v.35, p.325-336, 2011.

EMBRAPA - Empresa Brasileira de Pesquisa Agropecuária. Centro Nacional de Pesquisa de Solos. Manual de métodos de análise de solo. Rio de Janeiro: Embrapa Solos, 2.ed., 1997. 212p.

EMBRAPA - Empresa Brasileira de Pesquisa Agropecuária. Centro Nacional de Pesquisa de Solos (Rio de Janeiro, RJ). Sistema brasileiro de classificação de solos. Brasília: Embrapa Produção e Informação, 2006. 412p.

Ferreira, D. F. Sisvar: versão 4.6. Lavras: DEX/UFLA, 2003. Software.

Follet, R. F.; Schimel, D.S. Effect of tillage practices on microbial biomass dynamics. Soil Science Society of America Journal, v.53, p.1091-1096, 1989.

Gang, L. U.; Sakagami, K.; Tanaka, H.; Hamada, R. Role of soil organic matter stabilization of water-stable aggregates in soils under different types of land use. Soil Science Plant Nutrition, v.44, p.147-155, 1998.
Garcia, M. R. L.; Nahas, E. Biomassa e atividades microbianas em solo sob pastagem com diferentes lotações de ovinos. Revista Brasileira de Ciência do Solo, v.31, p.269-276, 2007.

Insam, H.; Domsch, K. H. Relationship between soil organic carbon and microbial biomass on chronosequences of reclamation sites. Microbial Ecology, v.15, p.177-188, 1988.

Karlen, D. L.; Mausbach, M. J.; Doran, J. W.; Cline, R. G.; Harris, R. F.; Schuman, G. E. Soil quality: A concept, defininition, and framework for evaluation (Aguest editorial). Soil Science Society of America Journal, v.61, p.4-10, 1997.

Letey, J. Relationship between soil physical properties and crop production. Advances in Soil Science, v.1.p. 277-294, 1985.

Moreira, F. M. S.; Siqueira, J. O. Microbiologia e bioquímica do Solo. Lavras: UFLA, 2006. 729p.

Nishi, E.; Tejerina-Garro, F. L.; Maia, C. B. Caracterização da cobertura vegetal remanescente e implicações na conservação da biota na bacia do ribeirão João Leite, Goiás, Região Centro-Oeste. Revista Brasileira de Cartografia, v.62, p.649-660, 2010.

Ocio, J. A.; Brookes, P. C. An evaluation of methods for measuring the microbial biomass in soils following recent additions of wheat straw and characterization of the biomass that develops. Soil Biology and Biochemistry, v.22, p.685694, 1990.

Perez, K. S. S.; Ramos, M. L. G.; Mc Manus, C. Carbono da biomassa microbiana em solo cultivado com soja sob diferentes sistemas de manejo nos Cerrados. Pesquisa Agropecuária Brasileira, v.39, p.567-573, 2004.

Silva, A. C.; Horák, I.; Cortizas, A. M.; Vidal-Torrado, P.; J. R.; Grazziotti, P. H.; Silva, E. de B.; Ferreira, C. A.. Turfeiras da serra do espinhaço meridional - MG. I - Caracterização e classificação. Revista Brasileira de Ciência do Solo, v.33, p.1385-1398, 2009.

Valladares, S. G. Caracterização de solos brasileiros com elevados teores de material orgânica. Magistra, v.20, p.95104, 2008.

Vance, E. D.; Brookes, P. C.; Jenkinson, D.S. An extraction method for measuring soil microbial biomass - C. Soil Biology and Biochemistry, v.19, p.703-707, 1987.

Weber, M. A.; Mielniczuk, J. Estoque e disponibilidade de nitrogênio no solo em experimento de longa duração. Revista Brasileira de Ciência do Solo, v.33, p.429-437, 2009. 